Conclusion These findings suggest that an early screening for lost of bone mass should be done, allowing the beginning of adequate therapy, in order to assure life quality to middle aged and older women.

\section{P1-229 DIET QUALITY OF MALE ADULT PARTICIPANTS HIM STUDY- BRAZIL (NATURAL HISTORY OF HPV INFECTION IN MEN): MULTICENTRIC STUDY}

doi:10.1136/jech.2011.142976e.22

J Carlos, A Previdelli, G Bartira, M L Baggio, A Giuliano, L Villa, E Lazcano, D Marchioni, * R Fisberg. University of Sao Paulo, Sao Paulo, Brazil

Introduction The study "Natural History of HPV Infection in Men" (HIM study) is an international multicenter prospective cohort study that seeks to determine the incidence, persistence and remission of human papillomavirus (HPV) infection in men.

Objective To assess the dietary quality on participants in HIMBrazil.

Methods Dietary intake of 70 participants were measured by two $24 \mathrm{~h}$ dietary recalls. The Brazilian Healthy Eating Index Revised (BHEI-R), developed according to current nutritional recommendations was used.

Methods The BHEI-R comprises a 12-components system of nine food groups based on Brazilian Dietary Guidelines (2006), which daily portions are expressed on energy density. Intakes at the level of the standard or more were assigned the maximum number of points: 5 to Total Grains; Whole Grains; Dark-Green and Orange Vegetables and Beans; Total Vegetables; Total Fruit and Whole Fruit); 10 to Dairy Products; Meat and Beans; Oils; Saturated Fat; and Sodium and 20 for SoFAAS (total calories from solid fat, alcohol and added sugar.

Results The mean B-HEIR score was 59.7 \pm 10.3 . For Total Grains

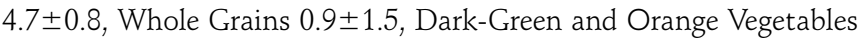

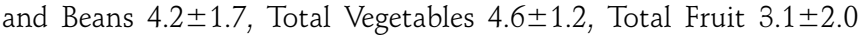
and Whole Fruit 2.9 \pm 2.3 , Dairy Products 4.7 \pm 3.1 , Meat and Beans

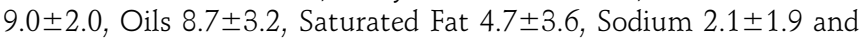
SoFAAS $10.2 \pm 5.4$

Conclusion It was observed a low BHEI-R score, reflecting the low consumption of whole grains, vegetables and fruits. Dietary modifications are necessary to achieve better quality on food intake, potencially beneficial to prevent immunodeficiency and susceptibility to infections.

\section{P1-230 VALIDITY OF SELF-REPORTED HYPERTENSION AMONG BRAZILIAN ADULTS}

doi:10.1136/jech.2011.142976e.23

S S de Castro Selem, M A de Castro, J V Carlos, B Gorgulho, D M L Marchioni, R M Fisberg, * C L Galvão. University of Sao Paulo, Sao Paulo, Brazil

Introduction Hypertension, a chronic disease with high prevalence and trend of increase in Brazil, has important influence on morbidity and mortality. Self-report are widely used to assess hypertension in surveys, due to low cost. However, there are few validation studies of the self-reported hypertension data.

Objective To investigate the validity of self-reported hypertension among adults.

Methods Data from a population-based survey and a complementary study carried out between 2008 and 2010, in 186 Brazilian adults (20 y or over), residents in São Paulo. Sensitivity, specificity as well as predictive values positive (PVP) and negative (PVN) of selfreported hypertension were calculated in relation to hypertension, according to the criteria of the Seventh Joint National Committee on Prevention, Detection, Evaluation, and Treatment of High Blood Pressure (means diastolic blood pressure $\geq 90 \mathrm{~mm} \mathrm{Hg}$ and/or systolic blood pressure $\geq 140 \mathrm{~mm} \mathrm{Hg}$ and/or present use of antihypertensive drugs).

Results The validity of self-reported hypertension was relatively high in adults: sensitivity $87 \%$, specificity $75 \%$, PVP $70 \%$ and PVN $89 \%$.

Conclusion Self-reported hypertension may be used in calculating the prevalence of this chronic disease for monitoring of hypertension trends, in the absence of measured blood pressure, among this population

\section{P1-231 FOOD INSECURITY IS ASSOCIATED WITH OVERWEIGHT IN PARIS METROPOLITAN AREA. AN ANALYSIS OF THE SIRS COHORT IN 2010}

doi:10.1136/jech.2011.142976e.24

${ }^{1,2} \mathrm{~J}$ Martin-Fernandez, ${ }^{1,2} \mathrm{E}$ Cadot, ${ }^{1,2} \mathrm{~F}$ Grillo, ${ }^{1,3} \mathrm{P}$ Chauvin. ${ }^{* 1}$ INSERM, U707, Research team on the social determinants of health and healthcare, Paris, France; ${ }^{2}$ UPMC Univ Paris 06, UMR-S 707, Paris, France; ${ }^{3}$ AP-HP, Hôpital Saint Antoine, Unité de santé publique, Paris, France

Background The relationship of food insecurity with overweight and obesity is still discussed in the literature. This work aimed to explore if this apparently paradoxical association was observed in Paris metropolitan area.

Methods We used data from the "Health, Inequality and Social Breakout" (SIRS) cohort, a longitudinal health and socio epidemiological, population based and representative survey of the general population of the Paris metropolitan area. This cross sectional analysis was based on the 2010 data. Participants' BMI was estimated using self-reported height and weight and computed in a dichotomous variable (BMI $<25$ vs $B M I \geq 25$ ). Food insecurity was estimated using the US HFSS and computed in a dichotomous variable: food secure vs food insecure (moderate/severe). Logistic regression models were estimated for men and women separately.

Results Overweight (BMI $\geq 25$ ) prevalence was $39.8 \%$. In men, nationality was significantly associated with being overweight: a European citizen has a higher risk of being overweight than a French one- $(\mathrm{OR}=2.89 ; \mathrm{p}=0.002)$. In women, socio professional group was a significant determinant of overweight, with a higher risk for workers' ( $\mathrm{OR}=5.37 \mathrm{p}<0.001$, ref $=$ Executives). After adjusting for age, nationality and socioprofessional group, food insecurity was associated with overweight in women $(\mathrm{OR}=2.24,95 \% \mathrm{CI}[1.32$ to $3.81])$ but this association was not significant for men $(\mathrm{OR}=1.54$, 95\% CI [0.83 to 2.86$]$ ).

Conclusion Food insecurity seems to be a stronger determinant of overweight among women. It remains important to explore and understand the pathway through which this situation is associated with overweight, particularly in terms of nutritional problems and food assistance programs.

\section{P1-232 HOSPITALISATION FOR CLINICAL COMPLICATIONS IN CANCER PATIENTS: ASSESSING THE NEED FOR PALLIATIVE CARE}

doi:10.1136/jech.2011.142976e.25

${ }^{1} \mathrm{~J}$ F Silva, ${ }^{2}$ Mattos, ${ }^{*}$ A P Silva. ${ }^{1}$ Mato Grosso do Sul State Office of Health, Campo Grande, Mato Grosso do Sul, Brazil; ${ }^{2}$ National School of Public Healthy, Oswaldo Cruz Foundation, Rio de Janeiro, Rio de Janeiro, Brazil; ${ }^{3}$ Department of Nursing, Federal University of Mato Grosso do Sul, Campo Grande, Mato Grosso do Sul, Brazil

Introduction Cancer is a public health problem in Brazil and the National Policy for Oncology determines that health services should 